\title{
CONTROLE DA COCHONILHA (Orthezia praelonga DOUGLAS, 1891) EM LARANJEIRA, COM INSETICIDAS GRANULADOS
}

\author{
F.A.M.MARICONI \\ Departamento de Zoologia-ESALQ/USP, C.P. 9 - CEP: 13418-900 - Piracicaba,SP. \\ L.L. NASS \\ CENARGEN/EMBRAPA-SAIN Parque Rural - CEP:70770-900 - Brasala,DF. \\ H.R. PASSOS; F. CINIGLIO NETO; F.M. VIEIRA JR.; H.M. CAMPOS NETO \\ Departamento de Zoologia-ESALQ/USP, C.P. 9 - CEP: 13418-900 - Piracicaba,SP.
}

RDSUMO: Com o objetivo de se avaliar a eficiencia de inseticidas granulados no controle da Orthezia praclonga em laranjeiras, foram empregados o aldicarbe $15 \%$ e o imidaclopride $5 \%$, aplicados ao solo. Os tratamentos foram seis, com quatro repetiçoes: A) testemunha; B) aldicarbe, $100 \mathrm{~g} / \mathrm{pl}$; C) aldicarbe, $65 \mathrm{~g} / \mathrm{pl}$; D) imidaclopride, $100 \mathrm{~g} / \mathrm{pl}$; E) aldicarbe, $130 \mathrm{~g} / \mathrm{pl}$; F) inidaclopride, 75g/pl. Foram feitas seis avaliaçoes: uma prévia e outras cinco apos 07,20 , 34, 49 e 70 dias da aplicação. Os melhores tratamentos foram: aldicarbe $100 \mathrm{~g} / \mathrm{pl}$ e aldicarbe, $130 \mathrm{~g} / \mathrm{pl}$, a0s 49 e 70 dias, respectivamente.

Describares: cochonilha, Orhezia praelonga, laranjeira, controle químico, inseticidas granulados

\section{CHEMICAL CONTROL OF COCCID (Orthezin preebange DOUGLAS, 1891) FOR ORANGE-TREES, WITH INSECTICIDE GRANULATED}

\begin{abstract}
ADSTRACT: The experiment was carried out on adult orange-trees in the county of Limeira, SP, Bravell. The objective was to evaluate the efficiency of insecticide granules with $15 \%$ aldicarb and $5 \%$ imidacloprid, applied to the soil, to control the citrus coccid Orthezia praelonga Douglas, 1891. Treatments were six: A) check; B) aldicarb, $100 \mathrm{~g} / \mathrm{pl}$; C) aldicarb, $65 \mathrm{~g} / \mathrm{pl}$; D) imidacloprid $100 \mathrm{~g} / \mathrm{pl}$; E) aldicarb, $130 \mathrm{~g} / \mathrm{pl}$; F) imidacloprid, $75 \mathrm{~g} / \mathrm{pl}$ of commercial insecticide granules. Six evaluations were made, one previous and other five 07, 20, 34, 49 and 70 days after application. The most efficient treatments were $\mathbf{E}$ and $\mathbf{B}$ at 49 and 70 days, respectively.

Key werls: citrus coccid, Orthezia praelonga, orange-tree, chemical control, granulated insecticides
\end{abstract}

\section{INTRODUÇÃO}

A citricultura, no Brasil, ocupa lugar de destaque pelo valor da exportação de suco concentrado, pelo comércio interno, e ainda, pela importância social, empregando grande número de pessoas. As pesquisas visam, portanto, amenizar os problemas da cultura, incluídos os relacionados ao ataque de pragas.

A cochonilha Orthezia praelonga é muito importante, pois diminui a produção. É cochonilha sem carapaça. Apresenta ovissaco onde são depositados os ovos. Vejamos alguns autores que trataram do assunto. COCKERELL (1900) foi o primeiro autor, no Brasil, a estudar a cochonilha; como "habitat" é citado o Pará, em Citrus limetta. HEMPEL (1900) descreveu o adulto feminino com numerosos detalhes; acrescentou a cidade de São
Paulo, em ramos de Hypts sp. ROBBS (1947) afirmou que a cultura citricola no ex-Distrito Federal (hoje, Rio de Janeiro) e no município fluminense de Nova Iguaçu vem sendo, nos últimos quatro anos, bastante prejudicada pelo inseto. $O$ ataque é evidenciado pela presença da fumagina. As medidas de combate preconizadas são eliminação de laranjeiras velhas, de baixa produtividade, poda de galhos, limpeza de troncos e galhos com escova de piassava, capinação em volta da planta atacada, caiação do tronco e pulverização de inseticidas (óleos miscíveis). ROBBS (1951) observou rápida proliferação do inseto em pomares submetidos à adubação com esterco animal e devido à condições climáticas favoráveis; que a formiga lavapé Solenopsis saevissima vive em simbiose com a ortézia e um fungo e dois insetos são inimigos. Pela primeira 
vez, um inseticida moderno (fosforado) é recomendado. GONÇALVES (1962) verificou que vários fungos reduzem a população da cochonilha. Diversos insetos predadores, a maior parte sem valor aparente, foram encontrados. PUZZI \& CAMARGO (1963) teceram comentários sobre a $O$. praelonga, principal praga dos citros na Baixada Fluminense, e sua possível introdução nos pomares de São Paulo. Apresentaram figura (climograma) da Universidade Rural do Rio de Janeiro (Baixada Fluminense) e as de Limeira, Ribeirão Preto, Pindamonhangaba e Ubatuba. Por comparação, verifica-se que a temperatura e a umidade relativa principalmente das três primeiras cidades, embora com médias de temperatura menores que as da Baixada Fluminense, sugerem a possibilidade do Vale do Paraíba (onde está Pindamonhangaba) e da região de Limeira, não dificultarem a multiplicacão do inseto, até nos meses mais frios. Sugerem, ain$\mathrm{da}$, que a cochonilha da Baixada Fluminense talvez pertença a uma raça que está se tornando progressivamente mais agressiva aos citros. CASSINO et al. (1975) aplicaram inseticidas fosforados sistêmicos sem diluição, segundo o método de aspersão tópica do tronco a uma altura de cerca de $15 \mathrm{~cm}$ do solo. A porcentagem de fêmeas mortas foi superior a $70 \% \mathrm{em}$ todos. Os inimigos naturais não foram atingidos. PAZINI et al. (1989) usaram produtos em pincelamento do tronco, no solo e em pulverização. Somente o dissulfotom granulado $5 \%$, à razão de $200 \mathrm{~g} /$ planta, controlou a cochonilha em níveis desejados. PINTO \& GRAVENA (1992) aplicaram inseticidas líquidos em pulverização e granulados no solo. A princípio, os pulverizados, principalmente $o$ fosalone, conduziram a melhores resultados contra as fêmeas adultas e ninfas; posteriormente, os granulados (aldicarbe $e$ imidaclopride) foram os mais eficientes.

\section{MATERIAIS E MÉTODOS}

Local: Campo experimental instalado na "Fazenda Panorama", pertencente ao Sr. Frederico C. Ivers, em Limeira, Estado de São Paulo.

Campo experimental: Plantas de laranja Lima (cavalo de laranja Caipira), com aproximadamente 23 anos de idade e plantadas no espaçamento de 7,0 × 6,0 m (238 plantas por hectare). A altura média das árvores variava de 4,5 a $5,0 \mathrm{~m}$. As plantas não tinham frutos, pois a colheita já fora realizada. As plantas estavam intensamente atacadas pelo inseto e cobertas por fumagina.
Tratamentos: Em número de seis, com quatro repetições (24 parcelas). Cada parcela experimental era constituída de uma planta, cercada por outras não tratadas. Foi adotado o método de blocos ao acaso. Os tratamentos, produtos comerciais, formulações e consumo de material podem ser vistos na TABELA 1.

Aplicacão: Foi efetuada em 12/09/1992, sendo os granulados colocados em dois sulcos laterais à planta, na projeção da copa, a uma profundidade de 5 a $8 \mathrm{~cm}$. A aplicação foi realizada sob chuva fina, mas o solo estava bem seco. Os granulados foram usados em dosagens mais altas que o normal, devido à altura das plantas.

Avaliacões: Foram realizadas seis avaliações, sendo uma prévia (02 dias antes da aplicação) e 07, 20, 34,49 e 70 dias após a aplicação dos inseticidas. Em cada uma das avaliações eram coletados, por parcela, em diferentes posições da planta, quatro pequenos galhos bem atacados pela cochonilha; entretanto, na última avaliação, foram coletados também quatro pequenos galhos ao acaso, estivessem ou não atacados. Em laboratório, as contagens das cochonilhas foram feitas somente nas três folhas apicais de cada galho, o que dava um total de doze folhas por parcela. Na última avaliação, foram feitas duas contagens: as das folhas colhidas de galhos atacados e a das folhas oriundas de galhos colhidos ao acaso. Os resultados das contagens encontram-se nas TABELAS 2 e 3.

Chuvas: Após um período de estiagem começaram as chuvas em setembro. Neste mês, até o dia da aplicação, caíram apenas $10 \mathrm{~mm}$. Depois da aplicação dos granulados caíram $91 \mathrm{~mm}$, ainda $\mathrm{em}$ setembro, $154 \mathrm{~mm}$ em outubro e $96 \mathrm{~mm} \mathrm{em}$ novembro (atE o dia 21, data do encerramento dos trabalhos).

Galhos internos: Os galhos situados fora do perímetro da copa, no interior da planta (ladrões), mantiveram vivas as colônias da cochonilha por período mais longo que as localizadas na copa. Acredita-se, por isso, que tais galhos devam ser eliminados antes do controle com granulados.

Reducão real ou eficiência: Calculada pela fórmula transformada de Abbott, que usa as porcentagens de sobrevivência da cochonilha (TABELAS 4 e 5). 
TABELA 1 - Combate à cochonilha ortézia em citros: tratamentos, formulações, concentrações e consumo de material. Limeira, 12/09/1992.

\begin{tabular}{lcccc}
\hline \multicolumn{1}{r}{ Tratamento } & \multicolumn{2}{c}{$\begin{array}{c}\text { Formulação Comercial e } \\
\text { Concentração de IA }\left(^{*}\right)\end{array}$} & Formulação & IA \\
\cline { 4 - 5 } & - & G $15 \%$ & - & - \\
\hline A - testemunha & Temik 150 & G 15\% & $65,0 \mathrm{~g}$ & $9,75 \mathrm{~g}$ \\
B - aldicarbe & Temik 150 & G $5 \%$ & $100,0 \mathrm{~g}$ & $5,00 \mathrm{~g}$ \\
C - aldicarbe & Premium 50 GR & G 15\% & $130,0 \mathrm{~g}$ & $19,50 \mathrm{~g}$ \\
D - imidaclopride & Temik 150 & G $5 \%$ & $75,0 \mathrm{~g}$ & $3,75 \mathrm{~g}$ \\
E - aldicarbe(**) & Premium 50 GR & & \\
F - imidaclopride & &
\end{tabular}

(*) $\mathrm{IA}=$ ingrediente ativo. $\mathrm{G}=$ granulado

(**) Tratamento padrão.

TABELA 2 - População de ninfas de cochonilha ortézia: avaliação prévia e no decorrer do experimento. Limeira, $10 / 09$ a 21/11/1992.

\begin{tabular}{lccccccc}
\hline \multirow{2}{*}{ Tratamento } & \multicolumn{7}{c}{ POPULAÇÃO MÉDIA DE NINFAS } \\
\cline { 2 - 8 } & Prévia & $\begin{array}{c}\text { Após } \\
\text { 07 dias }\end{array}$ & $\begin{array}{c}\text { Após } \\
\text { 20 dias }\end{array}$ & $\begin{array}{c}\text { Após } \\
\text { 34 dias }\end{array}$ & $\begin{array}{c}\text { Após } \\
49 \text { dias }\end{array}$ & $\begin{array}{c}\text { Após(*) } \\
70 \text { dias }\end{array}$ & $\begin{array}{c}\text { Após(**) } \\
70 \text { dias }\end{array}$ \\
\hline A - testemunha & 532,5 & 453,3 & 635,3 & 664,0 & 876,8 & 145,8 & 14,5 \\
B - aldicarbe & 526,8 & 362,8 & 295,8 & 283,0 & 111,8 & 27,5 & 0,0 \\
C - aldicarbe & 502,0 & 331,3 & 280,5 & 380,3 & 343,0 & 65,3 & 2,0 \\
D - imidaclopride & 536,0 & 406,8 & 414,3 & 549,3 & 754,0 & 51,5 & 1,5 \\
E - aldicarbe(**) & 516,0 & 324,3 & 250,3 & 303,8 & 112,3 & 1,8 & 0,0 \\
F - imidaclopride & 539,8 & 366,3 & 431,5 & 577,5 & 664,8 & 76,8 & 14,5 \\
\hline \hline
\end{tabular}

(*) Coleta de galhos atacados, como nas 5 anteriores.

(**) Coleta de galhos ao acaso.

Análise estatística: O número de cochonilhas das 4 parcelas, em todos os tratamentos e em cada avaliação, foi transformado em raiz quadrada de $x+0,5$, sendo $x$ a quantidade de cochonilha em cada parcela. A soma dos 4 valores assim transformados foi dividida por 4 , para se ter a população média do tratamento numa determinada avaliação.

Os resultados foram analisados pelo teste "F" e, a seguir, fez-se a análise das médias pelo teste de Tukey em nível de $5 \%$ de probabilidade (TABELAS 6 e 7 ).

\section{RESULTADOS E DISCUSSÃO}

Pelo exame das TABELAS 4 e 5 (mortalidades reais) e das TABELAS 6 e 7 (teste de Tukey em nível de 5\% de probabilidade), podese observar: 
TABELA 3 - População de adultos de cochonilha ortézia: avaliação prévia e no decorrer do experimento. Limeira, 10/09 a 21/11/1992.

\begin{tabular}{lccccccc}
\hline \multirow{2}{*}{ Tratamento } & \multicolumn{7}{c}{ POPULAÇÃo MÉDIA DE ADULTOS } \\
\cline { 2 - 8 } & Prévia & $\begin{array}{c}\text { Após } \\
07 \text { dias }\end{array}$ & $\begin{array}{c}\text { Após } \\
\text { 20 dias }\end{array}$ & $\begin{array}{c}\text { Após } \\
\text { 34 dias }\end{array}$ & $\begin{array}{c}\text { Após } \\
\text { 49 dias }\end{array}$ & $\begin{array}{c}\text { Após(*) } \\
\text { 70 dias }\end{array}$ & $\begin{array}{c}\text { Após }\left(^{* *}\right) \\
\text { 70 dias }\end{array}$ \\
\hline A - testemunha & 21,3 & 34,0 & 17,5 & 17,3 & 8,0 & 1,0 & 0,0 \\
B - aldicarbe & 26,0 & 29,8 & 15,5 & 7,8 & 2,5 & 0,0 & 0,0 \\
C - aldicarbe & 24,8 & 28,8 & 11,8 & 12,3 & 6,5 & 0,0 & 0,0 \\
D - imidaclopride & 23,5 & 35,5 & 12,3 & 20,5 & 10,5 & 0,3 & 0,0 \\
E - aldicarbe(**) & 18,5 & 31,3 & 8,3 & 9,5 & 0,0 & 0,0 & 0,0 \\
F - imidaclopride & 34,3 & 24,3 & 19,0 & 13,5 & 10,8 & 1,0 & $\mathbf{0 , 0}$ \\
\hline \hline
\end{tabular}

(*) Coleta de galhos atacados, como nas 5 anteriores.

(**) Coleta de galhos ao acaso.

TABELA 4 - Redução real (eficiência) na população de ninfas de cochonilha ortézia em cada tratamento, em cada avaliação. Limeira, 19/09 a 21/11/1992.

\begin{tabular}{lccccc}
\hline \hline \multirow{2}{*}{ Tratamento } & \multicolumn{5}{c}{ REDUÇÃo REAL DE NINFAS (\%) (*) } \\
\cline { 2 - 6 } & Após 07 dias & Após 20 dias & Após 34 dias & Após 49 dias & $\begin{array}{c}\text { Após 70 dias } \\
(* *)\end{array}$ \\
\hline A - testemunha & - & - & - & - & - \\
B - aldicarbe & 19,0 & 53,0 & 56,9 & 87,1 & 81,0 \\
C - aldicarbe & 22,4 & 53,1 & 39,3 & 58,5 & 52,6 \\
D - imidaclopride & 10,8 & 35,2 & 17,8 & 14,5 & 65,0 \\
E - aldicarbe & 26,2 & 59,3 & 52,8 & 86,8 & 98,9 \\
F - imidaclopride & 20,2 & 33,0 & 14,2 & 25,2 & 48,2 \\
\hline \hline
\end{tabular}

(*) Pela fórmula transformada de Abbott.

(**) Coleta de galhos atacados, como nas 4 anteriores.

Contagem prévia: tanto no caso das ninfas, como no dos adultos, as populações de todos os tratamentos se assemelham à testemunha.

$1^{\text {a }}$ Contagem (após 07 dias da aplicação): todos os tratamentos continuam semelhantes à testemunha nos dois casos.

$\underline{2^{a} \text { Contagem (20 dias): no caso das ninfas, os }}$ melhores são $E, B$ e $C$, que se assemelham entre si e diferem da testemunha. Para os adultos, todos os tratamentos são semelhantes entre si.

$3^{\text {a }}$ Contagem (34 dias): em relação às ninfas e adultos, todos os tratamentos não diferem da testemunha. E, B e C continuam os melhores em ambas as avaliações. 
TABELA 5 - Redução real (eficiência) na população de adultos de cochonilha ortézia em cada tratamento, em cada avaliação. Limeira, $19 / 09$ a 21/11/1992.

\begin{tabular}{lccccc}
\hline \hline \multirow{2}{*}{ Tratamento } & \multicolumn{5}{c}{ REDUÇÃo REAL DOS ADULTOS (\%) (*) } \\
\cline { 2 - 6 } & Após 07 dias & Após 20 dias & Após 34 dias & Após 49 dias & $\begin{array}{c}\text { Após 70 dias } \\
(* *)\end{array}$ \\
\hline A - testemunha & - & - & - & - & - \\
B - aldicarbe & 28,5 & 27,7 & 63,3 & 74,4 & 100,0 \\
C - aldicarbe & 27,4 & 42,4 & 39,0 & 30,0 & 100,0 \\
D - imidaclopride & 5,6 & 36,8 & 0,0 & 0,0 & 76,5 \\
E - aldicarbe & 0,0 & 45,9 & 36,7 & 100,0 & 100,0 \\
F - imidaclopride & 55,8 & 32,6 & 51,5 & 16,5 & 38,3 \\
\hline \hline
\end{tabular}

(*) Pela fórmula transformada de Abbott.

(**) Coleta de galhos atacados, como nas 4 anteriores.

TABELA 6 - Populações médias de ninfas de Orthezia praelonga (dados transformados em raiz quadrada de $x+0,5$ ) e resultados estatísticos (Tukey, $5 \%$ ).

\begin{tabular}{|c|c|c|c|c|c|c|c|}
\hline \multirow[t]{2}{*}{ Tratamento } & \multicolumn{7}{|c|}{ Médias das Populações de Ninfas e Resultados Estatísticos } \\
\hline & Prévia & $\begin{array}{c}\text { Após } 07 \\
\text { dias }\end{array}$ & $\begin{array}{c}\text { Após } 20 \\
\text { dias }\end{array}$ & $\begin{array}{c}\text { Após } 34 \\
\text { dias }\end{array}$ & $\begin{array}{c}\text { Após } 49 \\
\text { dias }\end{array}$ & $\begin{array}{l}\text { Ap6s }(*) \\
70 \text { dias }\end{array}$ & $\begin{array}{l}\text { Após(**) } \\
70 \text { dias }\end{array}$ \\
\hline A & 22,06 a & $21,02 \mathrm{a}$ & 25,12 a & 25,29 a & 29,51 a & $11,64 \mathrm{a}$ & $3,39 a$ \\
\hline B & 22,52 a & $18,63 \mathrm{a}$ & 16,45 b & $16,60 \mathrm{a}$ & $10,53 \mathrm{c}$ & $3,51 \mathrm{~b}$ & 0,71 a \\
\hline $\mathbf{C}$ & $22,41 \mathrm{a}$ & 18,13 a & $16,59 \mathrm{~b}$ & 19,11 a & 17,45 bc & $6,82 \mathrm{ab}$ & $1,41 \mathrm{a}$ \\
\hline D & $23,04 \mathrm{a}$ & 20,05 a & $20,29 \mathrm{ab}$ & 23,32 a & 27,42 a & $6,31 \mathrm{ab}$ & $1,28 \mathrm{a}$ \\
\hline $\mathbf{E}$ & 22,72 a & 17,82 a & $15,51 \quad b$ & $15,56 \mathrm{a}$ & $10,46 \mathrm{c}$ & $1,22 \mathrm{~b}$ & $0,71 \mathrm{a}$ \\
\hline $\mathbf{F}$ & $22,71 \mathrm{a}$ & 19,04 a & $20,42 a b$ & $23,03 \mathrm{a}$ & $25,10 a b$ & $7,03 \mathrm{ab}$ & $3,44 \mathrm{a}$ \\
\hline CV (\%) & 21,30 & 11,86 & 18,80 & 28,67 & 20,18 & 54,83 & 68,56 \\
\hline D.M.S. & 11,06 & 5,21 & 8,24 & 13,50 & 9,32 & 7,68 & 2,87 \\
\hline
\end{tabular}

Médias seguidas de mesma letra, não diferem estatisticamente entre si.

$\left.{ }^{*}\right)$ Coleta de galhos atacados.

(**) Coleta de galhos ao acaso.

$4^{\text {a }}$ Contagem (49 dias): contra as ninfas, E, B e C são os melhores, embora sejam semelhantes entre si. Para os adultos, $\mathrm{E}$ difere da testemunha, sendo o melhor tratamento. B e C, embora semelhantes à testemunha, têm destaque no controle.
5. Contagem (70 dias, coleta de galhos atacados): E e B diferem da testemunha e são os melhores no caso das ninfas. Para os adultos, todos os tratamentos são semelhantes d̀ testemunha, porém E, B e C são os melhores. 
TABELA 7 - Populações médias de adultos de Orthezia praelonga (dados transformados em raiz quadrada de $\mathrm{x}+0,5)$ e resultados estatísticos (Tukey, $5 \%$ ).

\begin{tabular}{cccccccc}
\hline \hline \multirow{2}{*}{ Tratamento } & \multicolumn{7}{c}{ Médias das Populações de Adultos e Resultados Estatísticos } \\
\cline { 2 - 8 } & Prévia & $\begin{array}{c}\text { Após 07 } \\
\text { dias }\end{array}$ & $\begin{array}{c}\text { Após } 20 \\
\text { dias }\end{array}$ & $\begin{array}{c}\text { Após } 34 \\
\text { dias }\end{array}$ & $\begin{array}{c}\text { Após } 49 \\
\text { dias }\end{array}$ & $\begin{array}{c}\text { Após(*) } \\
70 \text { dias }\end{array}$ & $\begin{array}{c}\text { Após(**) } \\
70 \text { dias }\end{array}$ \\
\hline A & $4,64 \mathrm{a}$ & $5,84 \mathrm{a}$ & $4,14 \mathrm{a}$ & $4,14 \mathrm{a}$ & $2,90 \mathrm{ab}$ & $1,15 \mathrm{a}$ & $0,71 \mathrm{a}$ \\
B & $5,06 \mathrm{a}$ & $5,44 \mathrm{a}$ & $3,54 \mathrm{a}$ & $2,76 \mathrm{a}$ & $1,56 \mathrm{ab}$ & $0,71 \mathrm{a}$ & $0,71 \mathrm{a}$ \\
C & $4,98 \mathrm{a}$ & $5,36 \mathrm{a}$ & $3,46 \mathrm{a}$ & $3,46 \mathrm{a}$ & $2,44 \mathrm{ab}$ & $0,71 \mathrm{a}$ & $0,71 \mathrm{a}$ \\
D & $4,83 \mathrm{a}$ & $5,81 \mathrm{a}$ & $3,51 \mathrm{a}$ & $4,45 \mathrm{a}$ & $3,22 \mathrm{a}$ & $0,84 \mathrm{a}$ & $0,71 \mathrm{a}$ \\
E & $4,29 \mathrm{a}$ & $5,25 \mathrm{a}$ & $2,94 \mathrm{a}$ & $2,55 \mathrm{a}$ & $0,71 \mathrm{~b}$ & $0,71 \mathrm{a}$ & $0,71 \mathrm{a}$ \\
F & $5,73 \mathrm{a}$ & $4,93 \mathrm{a}$ & $4,28 \mathrm{a}$ & $3,38 \mathrm{a}$ & $3,02 \mathrm{a}$ & $1,06 \mathrm{a}$ & $0,71 \mathrm{a}$ \\
\hline CV (\%) & 22,06 & 23,80 & 31,74 & 43,50 & 42,58 & 43,86 & - \\
D.M.S. & 2,50 & 2,98 & 2,66 & 3,45 & 2,26 & 0,87 & \\
\hline \hline
\end{tabular}

Médias seguidas de mesma letra, não diferem estatisticamente entre si.

(*) Coleta de galhos atacados.

(**) Coleta de galhos ao acaso.

6 Contagem ( 70 dias, coleta de galhos ao acaso): tanto para as ninfas, como para os adultos, todos os tratamentos são semelhantes à testemunha. No caso das ninfas, E e B são os melhores. Para os adultos, não houve diferença alguma.

\section{CONCLUSÕES}

No controle da cochonilha, os melhores tratamentos foram E e B, (respectivamente, $130 \mathrm{~g}$ e $100 \mathrm{~g}$ do granulado de aldicarbe por laranjeira). $\mathrm{D}$ e $\mathrm{F}$ (respectivamente, $100 \mathrm{~g} \mathrm{e} 75 \mathrm{~g}$ de granulado de imidaclopride por planta) não se salientaram.

\section{AGRADECIMENTOS}

Ao Sr. Frederico G. Ivers, proprietário da Fazenda Panorama e Eng ${ }^{\circ}$ Agr $^{\circ}$ Mauro Sagotti, da citada Fazenda, pelas facilidades concedidas.

\section{REFERENCIAS BIBLIOGRÁFICAS}

CASSINO, P.C.R.; COSTA, F.A.; DALCOMO, E.L.; RACCA FILHO, F. Contribuição para o controle integrado da Orthezia praelonga Douglas, 1891 (Homoptera-Ortheziidae) em citros spp. A Lavoura, Rio de Janeiro, v. 78, p.5-8, 1975.
COCKERELL, T.D.A. Notas sobre coccidas brasileiras. I. Uma lista de algumas coccidas coligidas pelo Dr. Emilio A. Goeldi, no Pará, Brasil. Revista do Musen Paulista, São Paulo, v.4, p.363-364, 1900.

GONÇALVES, C.R. Perspectivas de combate biológico às principais pragas das plantas cultivadas na Baixada Fluminense. Boletim do Instituto de Ecologia e Experimentaçāo Agricolas, Rio de Janeiro, n.21, p.65-78, 1962.

HEMPEL, A. As coccidas brasileiras. Revista do Museu Paulista, São Paulo, v.4, p.365-537, 1900.

PAZINI, W.C.; DIBELLI, W.; BUSOLI, A.C. Controle de Orthezia praelonga Douglas, 1891 (Homoptera, Ortheziidae) em citros, através de inseticidas aplicados no solo, no tronco e na copa. In: CONGRESSO BRASILEIRO DE ENTOMOLOGIA, 12., 1989. Belo Horizonte. Resumos... Belo Horizonte: SEB, 1989. p.371.

PINTO, R.A.; GRAVENA, S. Eficiência de inseticidas granulados e líquidos no controle de Orthezia praelonga Douglas, 1891 e Toxoptera citricidus Kirk. em laranjeira Citrus sinensis Osbeck. Jaboticabal: FCAV/UNESP, 1992. 26p. (Laudo TÉcnico). 
PUZZI, D.; CAMARGO, A.P. Estudo sobre a possibilidade da adaptação climática da Orthezia praelonga Douglas nos pomares de citros do Estado de São Paulo. O Biológico, São Paulo, v.29, n.5, p.81-85, 1963.

ROBBS, C.F. O "piolho branco" da laranjeira uma ameaça à citricultura do Distrito Federal. Boletim do Campo, Rio de Janeiro, v.3, n.19, p.1-4, 1947.
ROBBS, C.F. Principais cochonilhas das plantas cítricas na Baixada Fluminense. Boletim do Campo, Rio de Janeiro, v.7, n.41, p.5-13, 1951.

Recebido para publicação em 13.12.93

Aceito para publicação em 25.01.94 